\title{
Les contes de fées, ça c'est beau !!!!
}

Fairy tales, it's beautiful!!!!

\section{Nicole Belmont}

\section{(2) OpenEdition}

\section{Journals}

\section{Édition électronique}

URL : https://journals.openedition.org/clo/8142

DOI : $10.4000 /$ clo.8142

ISSN : 2266-1816

\section{Éditeur}

INALCO

\section{Édition imprimée}

Date de publication : 3 décembre 2019

Pagination : 173-200

ISBN : 9782858313716

ISSN : 0396-891X

\section{Référence électronique}

Nicole Belmont, «Les contes de fées, ça c'est beau !!!! », Cahiers de littérature orale [En ligne], 86 | 2019, mis en ligne le 03 décembre 2020, consulté le 22 mars 2022. URL : http://journals.openedition.org/ clo/8142 ; DOI : https://doi.org/10.4000/clo.8142

\section{(c) $(1)(8$}

Cahiers de littérature orale est mis à disposition selon les termes de la Licence Creative Commons Attribution - Pas d'Utilisation Commerciale 4.0 International. 


\title{
Les contes de fées, ça c'est beau !!!!
}

\author{
Nicole BeLmont \\ EHESS - Laboratoire d'anthropologie sociale (Collège de France)
}

Au début de son célèbre essai, Le Narrateur, Walter Benjamin invoque l'expérience journalière qui « nous dit que l'art de narrer touche à sa fin ». « Ne s'est-on pas aperçu à l'armistice que les gens revenaient muets du front ? non pas enrichis mais appauvris en expérience communicable ${ }^{1}$. $\gg$ W. Benjamin ne pouvait savoir qu'en France, depuis 1924, trois jeunes femmes s'efforçaient d'inventer, peut-être de réinventer, un art de conter à l'usage des enfants, ni qu'elles en avaient la possibilité matérielle grâce à des fonds provenant des États-Unis et destinés à doter de bibliothèques les « régions dévastées », dévastées par cette même guerre, la Grande.

La bibliothèque L'Heure joyeuse est inaugurée le 12 novembre 1924. Elle s'installe dans le quartier de Saint-Séverin, rue Boutebrie, dans le $5^{\mathrm{e}}$ arrondissement : proche du Quartier latin, il est plutôt populaire.

Le public de «L'Heure joyeuse », et c'est là à notre avis un de ses plus grands intérêts, est très éclectique. Filles et garçons appartiennent à des milieux très divers : enfants des classes prolétariennes, enfants des classes bourgeoises ; enfants étrangers, israélites pour la plupart, et dont les parents parlent à peine le français ; enfants des écoles de la ville de Paris, enfants des écoles privées, enfants des lycées ou des collèges catholiques, etc. ${ }^{2}$

1. BENJAMIN, 1995, p. 205.

2. LERICHE, 1934, p. 266. 
Conscientes de l'originalité de leur entreprise tout autant que de ses difficultés, elles ont pris soin de consigner jour après jour leur pratique en tant que bibliothécaires et en tant que conteuses, en leur accordant la même importance. Nous parlerons ici de leur activité de conteuses.

\section{Tout est à inventer}

Le nombre considérable de documents émanant des archives de L'Heure joyeuse et leur fragmentation rendent difficiles leur restitution et leur synthèse. Nous avons choisi de suivre une méthode en quelque sorte « régressive », partant d'une époque plus tardive, celle des années d'après-guerre où les deux conteuses toujours présentes ${ }^{3}$ ont été en mesure de maîtriser leur longue expérience, après l'avoir peu à peu, quasiment jour après jour, menée, précisée, affinée : celle où elles ont pu passer des courtes notations quotidiennes à l'exposé de la méthode. Alors que l'Heure du conte a repris en 1948, paraît en 1956 On raconte. Contes recueillis et présentés par Mathilde Leriche. Ce volume, essentiellement anthologique, sera réédité en 1969 sous le titre On raconte encore... proposant des références nouvelles et renvoyant à la première édition pour ce qui concerne l'histoire de l'Heure du conte et les conseils pratiques (choix, préparation, contage). L'ouvrage de Marguerite Gruny, $A B C$ de l'apprenti conteur (1987) se préoccupe de savoir ce qu'est le conte en général et ses usages pour les enfants, le projet est plus théorique. Elle propose cependant en dernière partie quatre récits, « histoires adaptées pour l'"Heure du conte" suivies de notes sur le travail de préparation ».

Ces premières bibliothécaires se révèlent être des conteuses hors pair. Jusqu'à leur retraite, elles manifesteront une exigence indéfectible dans le choix et la préparation des histoires lues ou racontées. C'est là l'expression du respect qu'elles n'ont cessé de manifester à l'égard des enfants ${ }^{4}$.

Que raconter aux enfants, comment raconter aux enfants, quel dispositif adopter, faut-il séparer « grands » et « petits »? Sur ce dernier point, on

3. Claire Huchet (1898-1993), directrice, quitte l'entreprise en 1929 : mariée, elle s'installe aux États-Unis. Marguerite Gruny (1903-1993) lui succède, assistée de Mathilde Leriche (1900-2000). Elles prendront leur retraite respectivement en 1968 et 1965. C. Huchet n'a pas été remplacée en tant que bibliothécaire-conteuse. Des stagiaires venaient pour s'initier au métier avec plus ou moins de succès.

4. Patte, 2005, p. 120. 
s'aperçoit que la frontière est poreuse. Des grands prévenus que c'est un conte pour les plus jeunes, se divertissent autant qu'eux à l'écoute du récit. Ou encore ils font montre d'indulgence amusée 5 . Autant de questions et bien d'autres encore qui se posent impérativement aux jeunes bibliothécaires, sans entamer leur détermination et en dépit de leur manque presque total d'expérience. Tout est à inventer ${ }^{6}$.

Leur méthode est empirique et expérimentale. Toute séance de contage sera consignée, de façon succincte il est vrai. Mais les éléments suivants sont considérés comme indispensables : date, nom de la conteuse, titre du « conte ${ }^{7} \gg$, nombre d'enfants (filles $v$ s garçons) et réception par cet auditoire inédit, sans oublier, éventuellement, la mention de quelque perturbation (sur ce point nous renvoyons à l'article de Marie-Christine Vinson dans ce numéro). Ces notations sont consignées sur des fiches bibliographiques.

\section{« Bienfaits des contes ». On raconte par Mathilde Leriche}

Ce recueil publié en 1956 se présente comme un manuel destiné à venir en aide aux bibliothécaires qui ne peuvent plus à cette date ne pas organiser de séances de contage et qui, souvent, n'ont reçu aucune formation pour s'y préparer. Plusieurs raisons justifient pour M. Leriche cette activité. Le contage favorise l'accès à la lecture personnelle des enfants désireux de retrouver ce qu'ils ont entendu, développant ainsi l'intelligence et formant le goût. Enfin...

5. Gruny, Heure du conte, 29 décembre 1938 : «Le château à l'ouest du soleil et à l'est de la lune, conte scandinave - plaît toujours aux plus petits. Les grands la trouvent jolie et l'écoutent avec plaisir. » Ou encore : « avec de jolis sourires » (M. Gruny, 18 décembre 1924).

6. Certes l'expérience de contage aux États-Unis remarquablement précoce (dès la moitié du XIX ${ }^{\mathrm{e}}$ siècle) représentait sinon un modèle pour la France - le contexte social et culturel étant tellement différent -, assurément un exemple à suivre. Claire Huchet avait découvert l'Heure du conte en faisant un stage à la Juvenile Court (San Francisco). De retour en France elle était devenue secrétaire de Mrs J.L. Griffith qui, en raison de ses compétences, l'avait placée à la tête de L'Heure joyeuse. Nous pensons que l'influence la plus importante - la plus efficiente - de la pratique américaine provient des deux volumes de Sara Cone Bryant qui propose à la fois des contes et des conseils de bon contage. Sur l'histoire de l'Heure du conte, en particulier américaine (Story House), on consultera avec profit l'article remarquablement documenté de G. Patte (2005).

7. L'appellation en quelque sorte « générique » veut qu'on appelle conte tout récit qui peut se dire oralement s'il suscite l'intérêt des auditeurs. 
Enfin, raconter ou lire des histoires aux enfants leur révèle la richesse des joies goûtées en commun. Ces jeunes êtres, différents les uns des autres, se trouvent tous dans l'attente d'une même émotion, la même inquiétude les étreint, le même rire les détend. [...] Communion bienfaisante. Un lien d'affection, de confiance, de reconnaissance unit les enfants à l'adulte dispensateur de ce bonheur. Lien délicat et précieux, amitié confiante qui permet de mieux comprendre et aider chaque enfant ${ }^{8}$.

Il ne faut pas méconnaître en outre « la saine et bonne gaîté » véhiculée par les contes : cette « détente collective [...] est comme une bouffée d'air frais qui purifie l'atmosphère ${ }^{9} \gg$. L'écoute collective se révèle ainsi socialisante : la lecture, solitaire quant à elle, risquerait-elle d'isoler l'enfant ? La lecture à haute voix où l'écoute est partagée serait-elle le moyen terme - idéal ou pis-aller ${ }^{10}$ entre les deux pratiques ? Elle a été largement utilisée à L'Heure joyeuse. Nous y reviendrons.

Autre point énoncé rapidement mais d'une grande importance et qui dévoile la totale maîtrise de cet art inédit jusque-là. Il faut au conteur de l' « honnêteté » : «Nous devons raconter pour les enfants, rien que pour eux ${ }^{11}$. Alors nait un courant de sympathie qui les unit au conteur ${ }^{12}$. $\gg$ Ils se sentent en effet respectés en tant que destinataires à part entière. Capables de ressentir « le sentiment de plénitude qui les pénètre à l'audition d'une belle histoire. [...] Ils apprécient son équilibre, jouissent des phrases bien frappées, des images poétiques ${ }^{13} \gg$. Il

\section{LERICHe, 1956, p. 12-13.}

9. Ibid., p. 13. Je remercie Agnès Clerc-Renaud de faire le lien entre les termes « saine et bonne gaîté », « purifie l'atmosphère » et l'introduction officielle de l' « hygiénisme » après la guerre, relayée dans le grand public. On lira plus loin à propos du rire suscité par une histoire drôle la comparaison avec de la « gymnastique », « un bain ou une promenade $\gg$.

10. «Une bonne lecture vaut mieux qu'une audition insuffisamment préparée. », Ibid., p. 12.

11. Le « double langage » est exclu. Pensons à Perrault qui propose des contes pour les enfants tout en y insérant nombres de clins d'œil vers les adultes de sa propre société : ces « récits à double-sens s'adressant à un public adulte imaginaire ou réel qui s'amuse ou s'attendrit aux dépens des enfants. », Ibid., p. 10.

12. Ibid., p. 10.

13.Ibid., p. 6. 
s'ensuit beaucoup d'obligations pour le conteur ${ }^{14}$. Il doit posséder un répertoire varié, abondant, sans cesse enrichi. Pas de spécialisation (comique, sentimental ou autre) : elle sature l'écoute et émousse l'intérêt. Le principe vaut pour la pratique elle-même. Si l'on raconte deux récits lors d'une même séance, ils doivent se mettre en valeur en s'opposant : une histoire « triste »/une histoire « drôle ». C'est un mauvais choix « sur le terrain » en 1931 qui permet de le comprendre :

Lucienne Jammes dit qu'elle aime mieux le « triste ». Mais Marie L. aimerait bien du « gai ». D’une manière générale, il nous aurait fallu une $2^{\mathrm{e}}$ lecture « gaie » après la première qui les a véritablement émus. Ils comptent bien sur une seconde lecture ${ }^{15}$.

Ont été racontés Le Loup, conte de Guy de Maupassant « qui a un vrai succès et qui est ce que Meunier et Lucienne Jammes préfèrent des deux », puis Comment le vicaire adjoint demanda en mariage la fille du pasteur [non identifié] : «(Calmes, mais moins d'intérêt. L. Jammes baille) ${ }^{16}$ ».

Cette lourde responsabilité qui pèse sur le conteur lui impose des obligations : « connaître le texte à fond », « retenir les expressions poétiques, celles qui font image $\gg$ et que, parfois, il est nécessaire d'apprendre par cœur ${ }^{17}$.

Pour posséder réellement l'histoire et la posséder à fond, nous ferons le travail d'analyse et de mémorisation plusieurs jours avant l'audition et nous la répéterons pour nous-mêmes avec soin ${ }^{18}$.

C'est ainsi que le conteur « récitera une histoire » comme disent les enfants, qui ressentent bien la différence entre langage courant et langage des contes ${ }^{19}$.

14. M. Leriche parle toujours du « conteur », heurtant la pratique qui témoigne d'une large prédominance féminine dans cette activité. Il faut y voir la désignation de la fonction à remplir plus que de la personne elle-même.

15. Dreyfus, Heure du conte, 6 février 1931.

16. Jeudi 18 mars 1928 : « Les jeunes lecteurs attendent avec impatience l'Heure du conte. Ce sera-t-il joli ? Ce sera-t-il rigolo ? Ce sera bientôt l'Heure du conte ? ( 9 ans). [...] Vous aimez les histoires drôles ? - Oh, oui ! Quand c'est triste j'ai envie de pleurer comme un veau ».

17. Rappelons ici la conception que se faisaient les conteurs canadiens étudiées par Vivian Labrie, qui voyaient le conte comme un itinéraire ponctué d'images fortes, de mises en scène quasi visuelles. LABRIE, 1978.

18. LERICHE, 1956, p. 10.

19. Geneviève Patte qui fit ses premières armes à L'Heure joyeuse parle, dans l'hommage qu'elle lui rend, « des textes qu'elle avait dits cent fois et qu'elle préparait toujours avec 
Elle aborde rapidement la question présente dans un grand nombre des notes prises par les conteuses - histoires racontées $v$ s histoires lues - et se prononce d'emblée pour les premières. En effet « un des grands charmes des histoires dites provient de ce qu'elles semblent jaillir du conteur ${ }^{20} \gg$.

Jusque vers dix ou onze ans une histoire dite apporte plus de joie qu'une histoire lue et le plus vif intérêt provoqué par une lecture n'atteint jamais « la magie de l'histoire racontée ». Un lecteur même expérimenté, ayant préparé avec soin son texte, demeure esclave du livre ${ }^{21}$.

La lecture de livres découpés en quatre ou cinq séances, voire huit, a été largement et tôt pratiquée à L'Heure joyeuse, mais réservée aux plus grands (11-15 ans). Les plus jeunes de leur côté ont besoin de l'immédiateté de la parole, sans que s'interpose le médiateur un peu rebutant qu'est le livre.

\section{Marguerite Gruny. La lecture racontée}

$A B C$ de l'apprenti conteur propose moins de récits en « prêt-à-porter » - il n’y en a que quatre - mais fournit une liste bibliographique d'ouvrages où puiser, répartis en six classes, parmi lesquelles (la seconde) : «Contes d'après des thèmes folkloriques très adaptés ». Ce sont tous des récits facétieux, populaires à l'origine certes, mais déjà passés par des réécritures altérantes. Il faudra longtemps avant que la littérature d'oralité seconde entre dans les bibliothèques pour enfants. On ne s'en étonnera pas, en particulier pour cette période de l'entre-deux-guerres. Entre 1870 et 1914 les collectes se font intensives dans la plupart des régions de France. Même publiées pour une grande part, elles sont restées confidentielles. La fin de cette guerre dévastatrice les rend obsolètes, un autre monde s'ouvre fait d'espoirs et de craintes. En outre l'apparence lacunaire de ces récits - ils ne disent pas tout, même les plus longs, et c'est là que réside leur efficacité symbolique est déroutante. Sans parler, mais c'est évident, de l'usage d'une langue parfois incorrecte. Les récits populaires reconnaissables que les conteuses sélectionnent

la même exigence », PATte, 2000, p. 15.

20. LERICHE, 1956, p. 12.

21. C'est nous qui soulignons. Elle utilisera une autre formule dans son article de 1934 : « Le conteur est libre tandis que la personne qui lit est liée ». Et un peu plus loin : « Le plus vif intérêt provoqué par une lecture n'a rien de comparable à la "magie" du conte. », LERICHE, 1934, p. 271. 
proviennent de recueils largement réécrits, voire réaménagés quant à leur trame narrative ${ }^{22}$. En dépit de leur oralité d'origine qui les désignerait d'emblée comme étant « bons à raconter »,

[...] les contes traditionnels nous paraissent néanmoins assez souvent un peu secs, comme inachevés, et d'une langue pauvre, malhabile. Mais quand un bon écrivain, séduit par leurs thèmes, s'en est emparé, les a relatés à sa manière, tout en restant proche de l'inspiration populaire et du style oral, celui qui veut les raconter n'a plus qu'à le suivre ${ }^{23}$.

La première rubrique bibliographique susceptible de fournir des « histoires » s'intitule « Contes d'auteurs divers » : conteurs assumés (les frères Grimm, Andersen) ou conteurs par goût (Georges Lenôtre, Gustave Flaubert). En troisième position viennent Les Mille et une nuits; en 4, les contes du Moyen Âge ; en 5 , les récits tirés de la mythologie grecque et romaine; en 6 , les romans à caractère « merveilleux ou légendaire à raconter par épisodes » (Carlo Collodi ou Selma Lagerlöf, largement mis à contribution en effet). On retrouve beaucoup de ces sources utilisées par les conteuses de l'Heure du conte durant cette période d'apprentissage, de recherche et de retours quotidiens sur leur pratique.

M. Gruny insiste longuement sur la préparation d'un conte, à commencer par le travail de mémorisation qu'elle considère plutôt comme une assimilation, excluant le mot-à-mot plein de dangers : trou de mémoire irrattrapable, récitation monotone ${ }^{24}$.

Il faut commencer par se préoccuper des faits essentiels dans leur enchaînement jusqu'à ce qu'on les voit se dérouler devant soi

22. On citera un exemple de ce dévoiement narratif provoqué par la réécriture « littéraire », provenant de l'un des quatre contes donnés par M. Gruny. Il s'agit de «L'Amour des trois oranges », revu et corrigé par Charles Deulin. Le prince qui ne veut pas se marier se décide ici à aller chercher une épouse après avoir ouvert nombre d'oranges envoyées à son père, car il a rêvé auparavant qu'il y trouvait une épouse. Le « réfecteur » anticipant cet épisode élimine ainsi, volontairement ou non, le motif éloquent de la malédiction de la vieille femme, figure maternelle menaçante, condamnant le prince à aller chercher au loin « l'Amour des trois oranges ».

23. GRUNY, 1987, p. 25.

24. «La délivrance de Fortuné, jeudi 17 mars 1932, Nous ne savons pas assez l'histoire pour en faire ressortir l'agrément littéraire : mais nous n'avons pas de "blanc" de mémoire et l'histoire est pleine de péripéties qu'ils suivent avec intérêt. » 
comme un film; en s'aidant au besoin d'un guide sur lequel on les aura consignés. ${ }^{25}$

Cette métaphore du conte remémoré comme film se déroulant devant le conteur durant sa performance complète le système exposé par les conteurs traditionnels canadiens évoqué à la note 16 : le conte merveilleux de tradition orale comme itinéraire à suivre, celui du héros ou de l'hérö̈ne, ponctué d'images et de mises en scène saisissantes, facilitant la mémorisation grâce à sa structure : itinéraire qui serait à la fois celui du héros ou de l'héroïne et celui que le conteur voit se dérouler devant lui.

Le travail de préparation est exigeant mais quelle récompense lorsque le conteur voit passer « l'histoire comme en reflet » sur le visage mobile des enfants. L'émotion des enfants durant l'écoute est la figure du contre-don de ceux-ci à la conteuse.

Très bonne séquence - attention profonde et sensible. La fin si belle les émeut beaucoup : ils restent silencieux un moment, comme suspendus; partis dans un autre monde ${ }^{26}$.

Elle explicite sa pensée quelque cinquante ans plus tard :

Et après, quand il [le conteur] a terminé ? Surtout pas de questions importunes venant de lui [...] après l'audition que chaque enfant puisse poursuivre son rêve et s'en aller avec ses propres impressions : que chaque enfant soit libre d'emporter la substance du conte comme il l'a comprise et de réfléchir à son gré sur ce qui l'a ému, amusé ou intrigué ${ }^{27}$.

À la circulation et au partage du récit succèdera la phase de rumination individuelle à la fois consciente et inconsciente. Une rêverie poétique productrice de force créatrice.

25. GRUNY, 1987, p. 29.

26. Gruny, Heure du conte, 30 mars 1933 : Le merveilleux voyage de Nils Olgersson à travers la Suède, épilogue.

27. LERICHE, 1956, p. 43. 


\section{Les années 1930}

L'entreprise ne semble pas susciter beaucoup d'écho à l'extérieur, sinon dans un cercle d'initiés. Nos bibliothécaires reçoivent des visiteurs intéressés, mais le monde de l'enseignement, « l'Instruction publique », reste indifférent et fermé. C'est le cas d'ailleurs bien des années plus tard comme le constate G. Patte :

Pour différentes raisons, l'exemple de L'Heure joyeuse est peu suivi, à Paris notamment. Lorsqu'à titre personnel, j'entreprends, au milieu des années 1960, la visite des bibliothèques de la capitale, je découvre que l'Heure du conte n'y est pas pratiquée ${ }^{28}$.

Nous avons retenu deux articles publiés l'un par Mathilde Leriche (1934) et l'autre par Marguerite Gruny (1938), issus pour le premier d'une « Causerie » donnée à la « Nouvelle Éducation » et pour le second d'une « leçon » donnée à la session intensive du Centre de formation des cadres de colonies de vacances à Nancy : des exposés à visée pratique dans des situations soit d'enseignement, soit d'encadrement.

Le nombre de colonies de vacances et centres de loisirs augmentant dans les années 1930 et particulièrement sous le Front populaire, leurs cadres ${ }^{29}$ ont besoin d'apprendre comment organiser des séances de contage, entre autres activités de loisirs. M. Gruny commence par affirmer sa conviction fondamentale concernant les enfants :

Nous les respecterons comme des esprits qui se cherchent et se forment, esprits malléables, gardant les empreintes, si légères soient-elles, de tout ce que nous leur distribuons, à qui nous devons donc donner ce qu'il y a de meilleur ${ }^{30}$.

Et non, ajoute-t-elle, comme de « charmants petits êtres incomplets ».

On trouve déjà dans ce texte beaucoup de conseils et de pratiques qui seront réaffirmés et développés dans son ouvrage de 1958, en constatant qu'à cette date la conteuse possède la totale maîtrise de sa pratique. À propos du choix des histoires, elle affirme que « les contes doivent être des œuvres d'art, œuvres bien construites, bien écrites, riches de poésie, d'une grande puissance dramatique

28. Patte, 2005, p. 123.

29. Des scouts et des éclaireurs interviennent à l'Heure du conte à un double titre : apprendre à conter et remplir des tâches d'encadrement.

30. GRUNY, 1938, p. 70. 
ou bien finement humoristiques ou d'un comique irrésistible ${ }^{31}$. » Ils se doivent également d'être moraux, ce qui écarte du répertoire Le Chat botté (Perrault) ou Le vaillant petit tailleur (Grimm). Cependant sont à exclure les contes dits moraux, « ceux qui sont des leçons de morale déguisées, et ont un air de sermon ». «Leçons de sagesse » assénées et non élaborées par chacun après l'écoute. Pour finir le choix des textes est facile : « retenir seulement les histoires qui vous plaisent », condition première pour réussir à faire passer l'émotion ou le comique ${ }^{32}$. «Certaines histoires sont si parfaitement écrites pour être racontées, le style en est si vivant, si direct, les événements s'y enchaînent avec une telle aisance » qu'elles ne demandent aucun travail d'adaptation. Elle cite « La table, l'âne et le bâton merveilleux » : on ne s'en étonnera pas puisqu'il s'agit d'un conte de tradition orale (« La serviette, l'âne et le bâton », ATU 563), un récit né dans l'oralité, modelé génération après génération pour être facilement mémorisable et transmissible.

En revanche un travail d'adaptation est à réaliser pour d'autres textes : élagage pour les Mille et une nuits, trop riche d'événements, de vocabulaire pour les contes français du $\mathrm{XVII}^{\mathrm{e}}$ siècle dont la langue est difficile. Mais on n'écartera pas « Le rossignol et l'empereur » d'Andersen dont le sens très profond risque - penserait-on - d'échapper aux enfants. Elle en convient, mais : «En communiquant un peu de votre propre émotion aux enfants, vous contribuerez à étendre, affiner leurs facultés émotives et leur intelligence ${ }^{33}$. 》 Après des recommandations quant à la pratique (longue préparation, diction, tenue, installation des auditeurs), elle fournit des listes de contes pour enfants de sept à douze ans : une liste d'histoires « ayant eu du succès à la Bibliothèque de "L'Heure joyeuse" », d'une part à raconter, de l'autre à lire. Une autre liste rassemble « de jolis contes qui ont du succès auprès des enfants et qui demandent peu d'adaptation ». Notons que, une fois de plus, Perrault ne s'en tire qu'avec deux titres : Cendrillon et La Belle au bois dormant.

\section{« Les histoires, mon vieux, c'est bien, mais faut que ça soit bien récité ! »}

Cette exclamation d'un enfant s'adressant à un camarade est rapportée par Mathilde Leriche dans un texte datant de 1934, « Causerie faite à la Nouvelle Éducation », relatant l'expérience de l'Heure du conte. La conteuse, bien

31. GRUNY, 1938, p. 72.

32. Ibid.

33.Ibid., p. 78 . 
convaincue de la justesse de cette opinion, tente ici de transmettre ce savoir-faire, acquis alors depuis dix années de pratique, à des auditeurs devant prendre en charge des enfants hors enseignement. Il ne s'agira, encore, que de la tranche d'âge de 6 à 11 ans, la plus difficile puisqu'elle requiert l'oralité intégrale, l'absence du livre, lequel s'interpose entre la conteuse et ses auditeurs, l'aliène en quelque sorte. Le texte commence par faire l'éloge du conte :

Le conte offre à l'enfant une détente; il est libre, nul contrôle. Le beau jardin est grand ouvert où il peut aller aussi loin qu'il veut, découvrir des trésors, faire une moisson pour lui seul, choisir librement ce qu'il désire ${ }^{34}$.

«Une grande puissance de vie émane du conte », ajoute-t-elle.

En outre c'est un art et une pratique qui s'apprennent. Aux États-Unis on y attache beaucoup d'importance. Les volumes de Sarah C. Bryant en témoignent. « Il y a une technique, des règles étudiées et expérimentées avec soin ${ }^{35}$. » On doit les apprendre comme on apprend à faire la classe ou à préparer un cours.

Ce texte manifeste fermement l'exigence de rigueur dans la pratique que réclame le respect que l'on doit à l'enfant, ce public qui « vient à nous de lui-même : c'est à nous de l'étudier, le comprendre, et lui donner ce qui lui convient $^{36} \gg$. Il est indispensable de varier le répertoire : par conséquent explorer sans trêve les ouvrages susceptibles de présenter des histoires bonnes à raconter ${ }^{37}$. «Comme le nombre de belles histoires n'est pas tellement grand nous éprouvons parfois de grosses difficultés pour renouveler notre répertoire ${ }^{38}$. »

Et ces histoires doivent alors être bien récitées. On y parviendra si l'on connaît parfaitement le récit, voire par cœur pour certains passages comme les expressions poétiques, les phrases drôles, les passages dramatiques. Les enfants savent apprécier le «bien-dire ». C'est ainsi que :

Un moment « L'Heure du conte » était désertée des amateurs car nous l'avions confiée aux stagiaires et l'une d'elles ne racontait

34. LERICHE, 1934, p. 262.

35. Ibid., p. 265 - c'est elle qui souligne.

36. Ibid., p. 266.

37. Voir dans ce numéro l'article de M.-F. Amara.

38. LERICHE, 1934, p. 267. 
pas bien ; les habitués n'allaient plus écouter les contes, disant « ce n'est plus cela ${ }^{39} !$ »

M. Leriche envisage ensuite l'organisation des séances de contage, en écho à leurs premiers « essais et erreurs ». Comment répartir histoires séparées et histoires en épisodes, « très aimées du public qui attend impatiemment d'un jeudi à l'autre ». Il faut tenir compte de la saison, « la grande vogue [souligné par M. L.] des contes est d'octobre à mai suivant que le printemps est plus ou moins précoce $^{40} \gg$. Dès qu'il fait beau, les enfants fréquentent plus volontiers les jardins publics. On réservera pour cette période des histoires qui ne se rattachent à aucun cycle.

« Le choix des histoires est donc un travail important auquel on ne saurait jamais donner trop d'attention ${ }^{41}$. » On doit également considérer l'installation des enfants : en demi-cercle et de façon à ce que la conteuse puisse voir tous les enfants qui, pareillement, pourront tous la voir ${ }^{42}$. Et bien entendu il faut connaître les ressources de sa voix, maîtriser les gestes qui doivent être expressifs. « Un geste esquissé, souligné par un changement de ton, est plus évocateur que des moulinets de bras. [...] Un bon moyen pour étudier son histoire est de la dire devant une glace ${ }^{43}$. » Les conteuses auraient, elles aussi, à passer par le stade du miroir ?

Ce texte touffu est à la fois éloquent et touchant. Il témoigne de toutes les difficultés et de tous les bonheurs de l'entreprise, alors que celui de Marguerite Gruny (1938), tout en étant en parfaite communauté d'idées, voire en complicité, est plus assuré, il se permet aussi un bilan positif. Il nous a semblé

39. LERICHE, 1934, p. 270.

40.Ibid., p. 268. Il a été souvent rapporté que les veillées traditionnelles pendant lesquelles on contait se déroulaient entre la Saint Martin (11 novembre) et l'équinoxe de printemps.

41.Ibid., p. 271.

42. Gruny, Heure du conte, $1^{\text {er }}$ décembre 1938 : « L'émotion est gâchée par la mauvaise installation - la conteuse ne voit pas une bande de quatre ou cinq petites filles (10-12 ans), habituellement gentilles, qui font les petites sottes aux passages les plus sentimentaux ce qui trouble l'atmosphère. » La perte du lien visuel altère la communication, il n'y a plus partage.

43. LERICHE, 1934, p. 271. 
important de les faire connaître l'un et l'autre pour témoigner de ces années d'apprentissage ${ }^{44}$.

\section{La tentation d'une île}

Nous nous permettons d'emprunter le titre d'un ouvrage paru en 2008, publié par un journaliste d'investigation, Philippe Bertherotte, intitulé plus exactement La Tentation d'une île. Derrière les caméras de l'Île de la Tentation ${ }^{45}$.

En avril 1928, du mardi 10 au vendredi 13, l'Heure du conte est consacrée à la lecture de l'ouvrage de Charles Vildrac, L'Île Rose, paru en 1924. Le timing est inhabituel : quatre jours de suite, du mardi 10 au vendredi $13^{46}$. Chaque séance dure entre une heure et une heure quinze. L'assistance est au nombre de quinze : 6 filles, 9 garçons. La lectrice est Mathilde Leriche. Le compte rendu concerne les quatre jours.

Sont pris dès la première page. - Ils sont tout à fait à leur aise, retrouvent des détails de leur vie d'écolier. Le merveilleux de l'île rose nouveau pour eux les enchante. - Ce sont des exclamations sans fin quelquefois inarticulées : Ah !!! Oh !!!... Il en a de la veine !!!... Si j'étais à sa place !!! Leurs yeux brillent, le temps passe sans qu'ils s'en aperçoivent. C'est une joie profonde et saine. Le grand avion, les canots automobiles, le chocolat du matin, etc., font pousser des cris de joie. Le soleil, la lumière, la joie qu'on sent à toutes les pages les pénètrent. La fuite de Tifernand pour aller voir sa mère malade cause une émotion profonde, on pleure : les Ah ! que c'est beau !!! ne tarissent pas. - le choix des noms si familiers et si jolis a beaucoup de succès. - Grand succès des illustrations : ils retrouvent tous les détails qui les ont charmés ${ }^{47}$.

\footnotetext{
44. On trouvera en annexe un passage de ce texte dans lequel M. Leriche compare deux écoutes du même ouvrage, qui fut un grand succès de lecture racontée en épisodes : Le merveilleux voyage de Nils Holgersson à travers la Suéde (1927 et 1933 respectivement). 45. Il s'agit d'une série de télé-réalité dont il montre les ressorts inconnus des téléspectateurs, en particulier la manipulation que subissent les participants.

46. Cette semaine suit le jour de Pâques de cette année 1928 : les enfants qui n'avaient pas quitté Paris pendant les vacances étaient libres tous les jours.

47. Leriche, Heure du conte, 10, 11, 12, 13 avril 1928.
} 


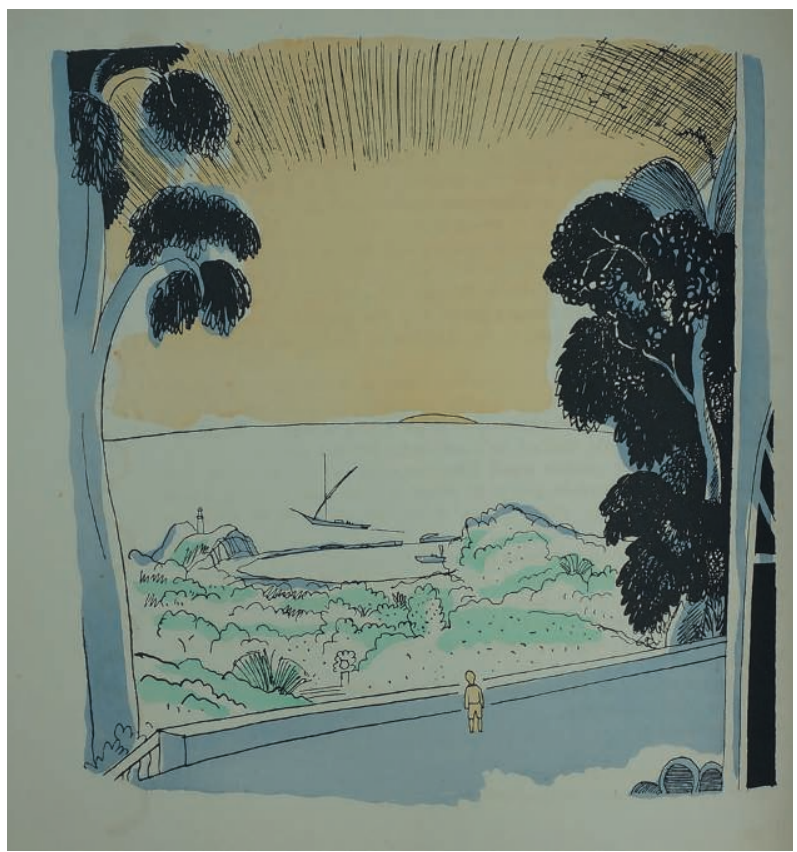

La terrasse

Edy-Legrand, illustration pour L'île rose, par Charles Vildrac, Paris, Tolmer, 1924 (exemplaire no 180/200), p. 116.

Fonds patrimonial Heure joyeuse, médiathèque Françoise-Sagan (Paris)

La conteuse conclut : « L'Île Rose, livre si plein de bonté joyeuse est une révélation. » Les enfants décident alors d'écrire à $\mathrm{C}$. Vildrac pour avoir la suite. C'est qu'en effet l'auteur, à la fin de son récit, laisse entendre qu'il pourrait s'intéresser de nouveau à ses personnages.

Samuel demande si on va bientôt avoir réponse à la lettre envoyée à C. Vildrac et la suite de L'Île Rose. Rarement un livre a plu à un tel point à des enfants. Leur souvenir leur revient sans cesse. C'est un souvenir plein de soleil et de lumière ${ }^{48}$.

La Colonie, suite de L'île rose, paraît en 1930, mais n'entre pas dans le patrimoine narratif de l'Heure du conte en dépit de la carte illustrée d'un bateau

48. Leriche, Heure du conte, jeudi 10 mai 1928. 
à laquelle l'auteur a répondu tardivement par une carte postale représentant Saint-Tropez, «Un coin du Vieux port. Quartier des pêcheurs », datée du 28 mars $1931^{49}$.

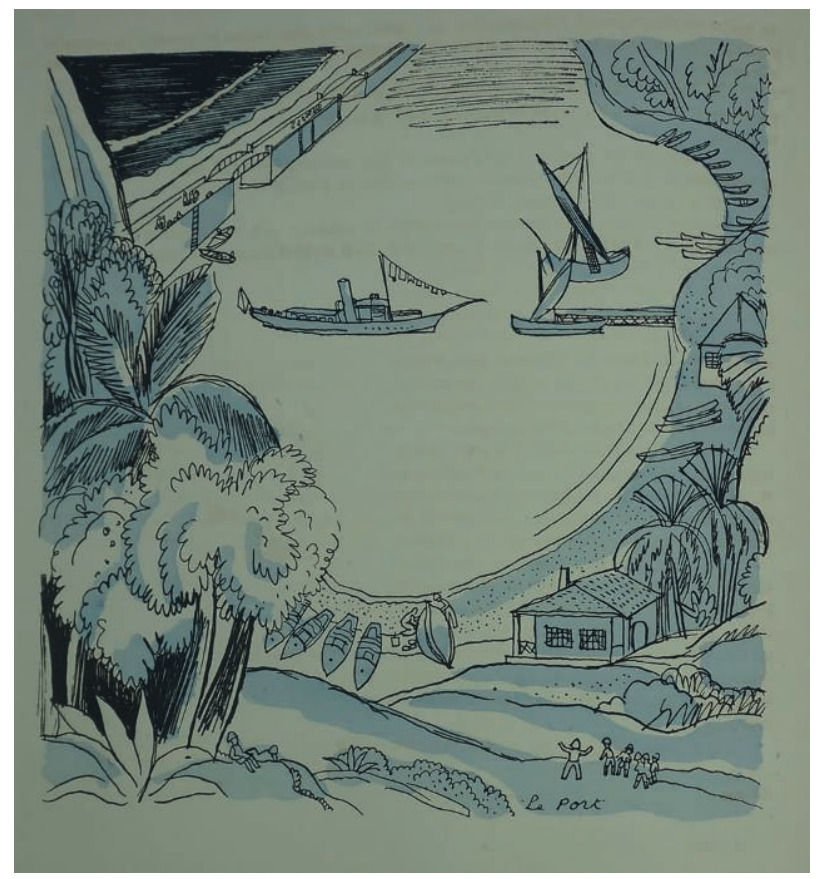

Le port de l' ̂̂le Rose

Edy-Legrand, illustration pour L'île rose, par Charles Vildrac, Paris, Tolmer, 1924 (exemplaire $\mathrm{n}^{\circ} 180 / 200$ ), p. 84.

Fonds patrimonial Heure joyeuse, médiathèque Françoise-Sagan (Paris)

Moins d'un an après ces premières séances, L'Île Rose est de nouveau présentée en lecture racontée et suivant le protocole normal : découpée en plusieurs séquences données jeudi après jeudi, du 7 au 28 février 1929. La conteuse est toujours M. Leriche. L'auditoire, beaucoup plus nombreux, restera remarquablement stable à chaque séance : 40 enfants dont 16 filles et 24 garçons.

49. C. Vildrac dit se trouver alors à Saint-Tropez, « un petit port de la méditerranée où tout ce que j'ai vu, depuis des années que j'y viens, m'a beaucoup servi pour écrire le « Colonie ». Je suis allé souvent à la pêche comme M. Lucas et Tifernand. » 
Ainsi que l'âge moyen : 11 ans environ ${ }^{50}$. Après la première séance, la conteuse note :

Sont pris tout de suite par le récit et profondément intéressés. - Retrouvent avec joie des détails de leurs vies d'école et s'exclament! Sont intrigués et se demandent quelle va être la suite. Ils essaient d'interroger la bibliothécaire. (Chap. 1 à 3) ${ }^{51}$.

Et à la suivante :

Leur intérêt et leur enthousiasme croissent. Ils vivent avec Tifernand. L'enchanteur les intrigue beaucoup. Ils sont très curieux de connaître la suite. Les détails choisis avec un art parfait les ravissent (la tenue d'aviateur par ex.). Ils se demandent quelles choses extraordinaires on va voir à l' ̂̂le Rose. (chap. 3 à 6)

Le commentaire de la troisième lecture est plus rapide, il dit l'attente anxieuse : « Ils sont aussi à l' ̂̂le Rose. Ils attendent avec impatience le passage tragique qu'ils pressentent. »

Le dénouement suscite toujours autant d'intérêt ${ }^{52}$ :

Angoisse et émotion. Ils sont haletants. Détente quand Tifernand est sauvé ; ravissement jusqu'à la fin. [...] Après la lecture ils regardent les illustrations et retrouvent avec plaisir les personnages et les paysages. Ils voudraient la suite.

La conteuse remarque très justement : « Le merveilleux nouveau pour eux les enchante, ils pourraient y être à la place des enfants, s'ils pouvaient quitter l'école. »C'est en effet un merveilleux « contemporain ». L'identification héroïque est facilitée puisqu'on leur présente des personnages qui leur ressemblent, qui vivent également une vie médiocre entre la famille, l'école, les amis, et qui pourraient se retrouver « entre soi »dans un lieu plein de lumière et de soleil. Le merveilleux ne relève plus d'un temps « intemporel » («il était une fois »), mais de la contemporanéité : il serait possible de s'y glisser.

50. Le succès de la première lecture durant les vacances de Pâques a sans doute incité les conteuses à la reprendre l'année suivante en respectant alors le rythme hebdomadaire.

51. Leriche, Heure du conte, 7,14, 21, 28 février 1929.

52. Mis à part une difficulté, récurrente d'ailleurs, quant à l'installation des enfants : ils sont si serrés qu'ils se gênent mutuellement. 


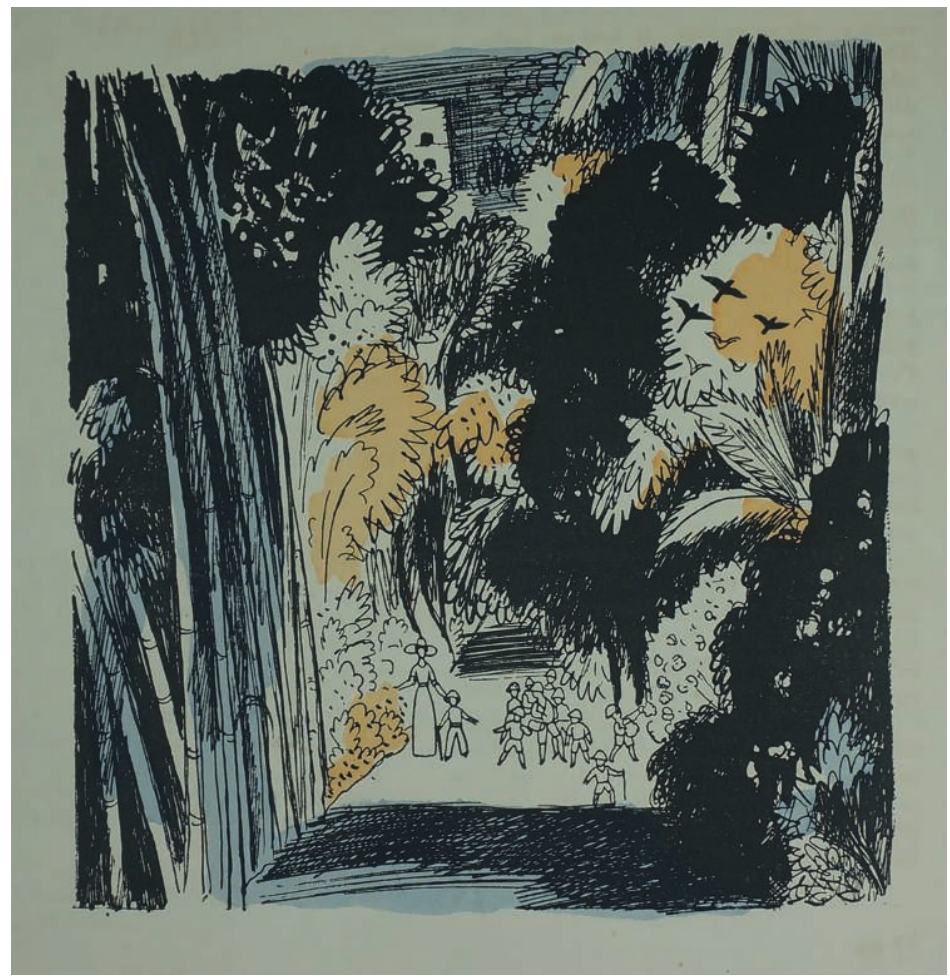

«Sur le sentier avec $\mathrm{M}^{\text {elle }}$ Gentil »

Edy-Legrand, illustration pour L'île rose ${ }^{53}$, par Charles Vildrac, Paris, Tolmer, 1924 (exemplaire no 180/200), p. 146.

Fonds patrimonial Heure joyeuse, médiathèque Françoise-Sagan (Paris)

L'ouvrage plébiscité par les enfants est l'œuvre d'un écrivain oublié de nos jours, mais reconnu en son temps (1882-1971) par un cercle d'écrivains et de poètes (comme Max Jacob ou Paul Éluard). Il est incité à écrire ce premier ouvrage destiné aux enfants par son illustrateur et ami Edy-Legrand ${ }^{54}$. Utopie,

53. Edy-Legrand construit cette image à la manière de Gustave Doré lorsqu'il illustre le Petit-Poucet : « en marchant il avait laissé tomber... » : les enfants sur un chemin étroit dans une forêt profonde, mais celle-ci est méditerranéenne.

54. Nous renvoyons au très intéressant article de Martine Jacques consacré à ces deux ouvrages de C. Vildrac, 2013. 


\section{CAHIERS DE LITTÉRATURE ORALE}

robinsonnade, ce roman raconte le séjour dans une île merveilleuse (inscrite sur peu de cartes géographiques !) d'un garçon habitant le faubourg Saint-Antoine, Tifernand, dont la vie se déroule entre sa famille (pauvre - son père, ouvrier, fabrique des caisses d'emballage - mais très affectueuse), l'école (où il connaît des déboires et des joies) et quelques amis. Il est repéré par un personnage étrange, M. Vincent qu'il nomme l'Enchanteur, un Merlin des temps modernes. Celui-ci l'emmène dans son domaine, une île aux rochers roses, au large de Marseille. On y accède grâce à l'avion de science-fiction qui permet à l'Enchanteur de s'y rendre rapidement et de se poser sans avoir besoin de piste d'atterrissage, comme le ferait un hélicoptère.

C'est un univers clos, dont tous les dangers physiques ont été enlevés, où toutes les contraintes ont disparu, où les adultes sont réellement au service des enfants, avec nourriture, vêtements, loisirs, éducation à volonté et en liberté totale. L'amitié et la bonne humeur y règnent, la concorde et la joie de vivre, chacun pensant aux autres, hors de souci du monde extérieur ${ }^{55}$.

Mais un univers entièrement masculin, sinon $\mathrm{M}^{\text {elle }}$ Gentil, l'institutrice des petits (au-dessous de 10 ans) et un univers interdit aux membres de la parenté. Le drame surgira de là. Tifernand apprend, alors qu'on le lui cachait, que sa mère est malade. Il essaie de revenir à Paris, mais il essuie une tempête entre l' ̂̂le Rose et Marseille, où il frôle la mort. Le drame infléchit la rigueur de l'Enchanteur qui organise une visite de Tifernand à sa famille et mieux encore, propose d'accueillir celle-ci durant les vacances d'été à l'Île Rose - y compris sa petite sœur Rirette et sans oublier les copains Bouboule et Théo. 


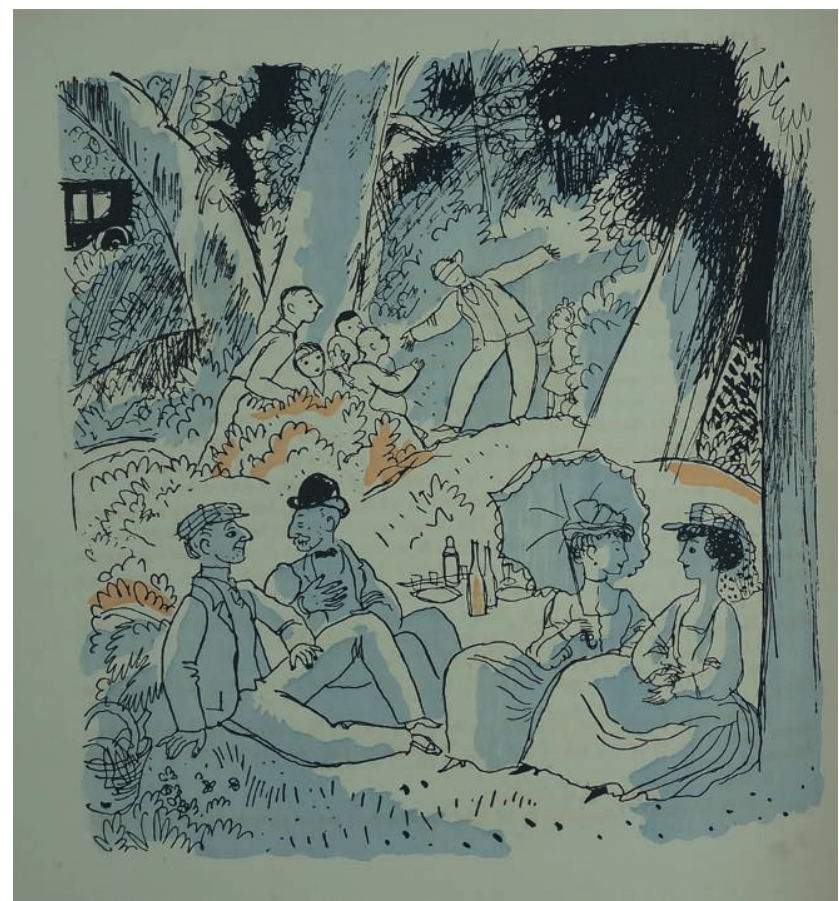

Le déjeuner sur l'herbe dans la forêt de Fontainebleau

Edy-Legrand, illustration pour L'île rose ${ }^{56}$, par Charles Vildrac, Paris, Tolmer, 1924 (exemplaire no 180/200), p. 146.

Fonds patrimonial Heure joyeuse, médiathèque Françoise-Sagan (Paris).

L'Enchanteur se trouve donc obligé d'infléchir les principes qui gouvernent le modèle utopique, remarque Martine Jacques, et qui, dans ce cas, se révèlent quasiment totalitaires.

Chez Vildrac, l'enchanteur utopique est aussi ambigu que son nom. L'enchantement peut désigner un moment de bonheur mais aussi une prise de possession magique d'autrui. Ses motivations (comme la jalousie qu'il manifeste lorsque Tifernand baptise son petit navire du nom de sa jeune sœur) et plus encore ses procédés

56. Edy-Legrand s'inspire ici du « Déjeuner sur l'herbe » d'Edouard Manet, l'esprit est moins provocant, mais la gravure est construite comme le tableau. 
(mensonge, rétention de cadeaux ou d'informations pour l'éloigner de sa famille) sont bien souvent négatifs : ils conduisent Tifernand à risquer la mort pour retrouver les siens. L'enfant ne veut pas céder à l'exigence totalitaire de l'utopie qui veut que l'on se dépouille du vieil homme qui est en soi ${ }^{57}$.

Les bibliothécaires de L'Heure joyeuse auraient-elles eu la tentation d'en faire un lieu d'entre soi, fonctionnant de manière étanche par rapport au monde extérieur, et particulièrement la famille des enfants, d'où viennent et où repartent les enfants chaque jour?

L'île est un espace globalement clos [...] qui impose son rythme et sa cohérence et qui exige de ses habitants un parfait respect de règles jugées suffisamment sages pour qu'elles soient naturellement respectées ${ }^{58}$.

Les bibliothécaires-conteuses ont-elles eu la tentation d'un lieu réservé à une élite se pliant aux règles démocratiques, mais, dans la réalité quotidienne, mal acceptées par certains ? En témoigne largement l'article de Marie-Christine Vinson dans ce numéro. Les manquements à l'ordre sont punis d'expulsions, de quinze jours parfois. La porte qui donne accès à ce lieu insulaire se doit d'être fermée et de s'ouvrir aux seuls qui acceptent de ne pas troubler ce huis-clos, cet entre soi rassurant, mais quelque peu élitiste. Un lieu étanche même par rapport à la famille ${ }^{59}$.

Serait-il possible d'expliquer par-là pourquoi la suite de $L$ 'Île Rose n'apparaisse pas dans le répertoire des lectures racontées de nos conteuses, en dépit de l'attente des enfants ? La Colonie débute avec la ruine de l'entreprise utopique, puis raconte son insertion dans un univers où l'on doit travailler pour gagner sa

57. JACQUES, 2013, p. 24.

58.Ibid., p. 7.

59. On rapportera rapidement un désaccord avec la mère d'une petite fille qui venait chercher celle-ci avant que débute l'Heure du conte : «Après elle me fait des cauchemars ! ». Pour les conteuses cette irruption du monde extérieur dans leur communauté « insulaire » était mal venue et non fondée. « [...] La petite fille ne se libérait-elle pas d'une protection excessive ? et la mère ne souffrait-elle pas d'une jalousie instinctive devant l'accueil enthousiaste que nous réservaient les enfants de cette garderie ? (rapporté par GRUNY, 1987, p. 22. L'Heure du conte avait lieu à cette époque dans le hall de l'école de la rue St Jacques). Le protocole de l'Heure du conte a le pas sur l'autorité familiale. 
$v^{6 i}{ }^{60}$. Les pouvoirs d'enchanteur de M. Vincent provenaient entièrement de son immense fortune qu'il vient de perdre, engloutie « comme un navire qui sombre dans la tempête ${ }^{61}$. » Comment ne pas penser alors au krach mondial de $1929^{62}$ ?

Il est rare que des échos d'événements politiques affleurent dans les notes d'après contage des deux bibliothécaires ${ }^{63}$. Le 8 octobre 1928, c'est dans le journal quotidien de la bibliothèque que $\mathrm{M}$. Gruny note la visite de Daniel P. lequel a eu un accident en Italie où il était en vacances avec ses parents. À Aoste, ils se sont trouvés devant un défilé de cinq cents personnes en chemise noire accompagnant un enterrement fasciste. Voulant prendre des photos, ils sont violemment agressés et voient leurs appareils confisqués. Ils restent surveillés jusqu'à leur sortie du territoire italien.

\section{«Les contes de fées, ça c'est beau ${ }^{64} ! ! ! ! ~ »$}

Le 12 avril 1934, les conteuses racontent La petite souris grise de Mme de Ségur. Les enfants restent « assez indifférents ». Elles décident alors de se lancer dans une « histoire inventée par elles », mettant en scène une famille d'éléphants venue passer quelques jours à Paris.

Ils se tordent de rire et poussent des cris de joie. Impression qu'ils ont besoin de rire et ont besoin d'une détente dans toute leur vie étriquée. C'est un besoin physique chez eux : comme de la gymnastique, un bain ou une promenade.

60. L'Enchanteur précise bien, dans le discours qui clôt l'ouvrage, qu'il s'agit de produire seulement en fonction de leurs besoins sans chercher à accumuler les richesses. VILDRAC, 1930, p. 246-248.

61. Ibid., p. 61.

62. En 1938 Hergé publie L'île noire, que l'on a envie de considérer comme le contre-modèle de L'Île Rose : lieu maléfique cachant les agissements d'une organisation criminelle internationale. Il est même question d'un avion, mais celui-ci s'écrase au sol.

63. Citons cependant : « 8 février 1934. - « Le Moine mystérieux » (conte A. Wahlenberg). M. Leriche - Sont très pris par l'histoire jusqu'à la fin. Ce jeudi n'a guère une atmosphère aux histoires les enfants étant excités par les événements et les troubles politiques. - Néanmoins ils sont très contents de leur séance.

64. « Les garçons ont été bien souvent les hardis défenseurs des fées, ils furent catégoriques et souvent émouvants : il fallait des fées, il fallait du merveilleux. D'instinct ils savaient la valeur symbolique et poétique des contes. », LERICHE, 1956, p. 14. 
Dans ce texte contemporain, M. Leriche parle de saine et bonne gaîté ${ }^{65}$.

Qui a vu rire des enfants auxquels on raconte une histoire drôle comprendra tout de suite la valeur de cette gaîté ! Il fait bon voir rire des enfants, rire à «se tordre », rire à pleurer. C'est comme une bouffée d'air frais qui leur arrive ; c'est un peu comme s'ils avaient pu courir et sauter ${ }^{66}$.

Les contes ne procurent pas seulement une détente physique par le rire. Ils provoquent également de l'émotion : c'est là le principal objectif des conteuses.

Ils écoutent tous sans un mouvement, les yeux fixés sur nous, sans un mouvement. Ce genre d'histoire simple et classique leur plaît infiniment. Un s'écrie : «Les contes de fées, ça c'est beau $^{67}$ !!!!»

Le récit raconté « les yeux dans les yeux » à un effet quasiment hypnotique. C'est un voyage dans une île rose, mentale celle-ci, qui apporte aux enfants une évasion loin de leur vie quotidienne difficile pour beaucoup.

Le signe qui ne trompe pas sur la qualité de l'émotion provoquée par l'écoute, c'est le silence qui suit. On pourrait multiplier les occurrences de ces notations après-coup : «Grande émotion. Quelques lecteurs restent quelques instants sans parler $^{68} \gg ;$; Grand intérêt et réelle émotion. Un long silence à la fin ${ }^{69} \gg ; \ll$ Et que de choses contient le silence profond qui suit les derniers mots du conte ${ }^{70} ! \gg$ Ces notations de la première heure se retrouvent tout au long de leur activité (jusqu'en 1939). Lors des lectures racontées, l'émotion grandit à chaque séance. Le premier stade, c'est « être pris ». Ainsi pour Pinokio $[\text { sic }]^{71}$.

Sont dans l'histoire ${ }^{72}$

65. LERICHE, 1934, p. 263. C'est elle qui souligne.

66. Ibid.

67. Leriche, 13 avril 1933.

68. Huchet, Heure du conte, 13 novembre 1924.

69. Ibid., 20 novembre 1924.

70. Ibid., 27 novembre 1924.

71. Raconté entre le 13 novembre et le 11 décembre 1930 par M. Leriche. Souvent repris, c'est un des succès de l'Heure du conte.

72.Ibid., 13 novembre 1924. 
Sont de plus en plus pris - Silences lourds d'inquiétude ${ }^{73}$

Public qui réagit vivement, vibre à toutes les aventures ${ }^{74}$

Exclamations désespérées et gémissements. [...] Silence lourd ${ }^{75}$.

La fin les ravit. Ils éprouvent un soulagement immense et une satisfaction profonde ${ }^{76}$.

Et concernant un autre des grands succès de ces lectures, Le Merveilleux Voyage de Nils Olgersson à travers la Suède (ci-dessous l'épilogue de cette série où il semble que le public n'était pas à la hauteur du texte, voir Annexe 1) :

Très bonne séance ; attention profonde et sensible. La fin si belle les émeut beaucoup ; ils restent silencieux un moment, comme suspendus; partis dans un autre monde ${ }^{77}$.

En outre, après les images mentales que chacun garde en mémoire, vient souvent la confrontation avec les images visuelles : les illustrations de l'édition italienne de Pinokio qui font revivre leurs émotions. « L'image de Pinokio devenu petit garçon les enthousiasme : «Oh ! qu'il est joli !». À ce surplus d'incitation émotive par l'image, fréquent lorsque la bibliothèque possède une édition illustrée, M. Gruny aurait aimé ajouter l'incitation musicale lorsqu'elle raconte Le Vaisseau fantôme, un arrangement personnel du livret de l'opéra de Wagner.

Beaucoup de succès. Ce conte est d'un grand effet dramatique. Le sacrifice de Senta dont le sens dépasse certainement la majorité des auditeurs semble pourtant les émouvoir profondément ${ }^{78}$.

Elle regrette que la bibliothèque ne possède pas de phonographe, certains airs auraient ajouté à l'émotion déjà forte.

73. Huchet, Heure du conte, 20 novembre 1924.

74. Ibid., 27 novembre 1924.

75. Ibid., 4 décembre 1924.

76. Leriche, Heure du conte, 11 décembre 1930.

77. Ibid., 30 mars 1933.

78. Gruny, Heure du conte, 8 décembre 1938. 
Ce n'est pas non plus la seule fois où les conteuses pensent que le récit puisse échapper, en partie du moins, aux enfants : ainsi pour « Le rossignol et l'empereur » ou « La petite sirène » d'Andersen et donc « le Vaisseau fantôme ». Peu importe : le récit laissera toujours une trace en eux. Ne pas comprendre totalement n'empêche pas l'imprégnation émotive.

Le silence après l'écoute respecté par les conteuses qui en conçoivent toute l'importance, anticipe ce que W. Benjamin disait du narrateur dans le texte cité au début de cette étude :

Car c'est le fait du narrateur né que de débarrasser une histoire de toute explication. [...] L'extraordinaire, le merveilleux, on le raconte avec la plus grande précision, mais on n'impose pas au lecteur l'enchaînement psychologique des événements. On le laisse libre d'interpréter la chose comme il l'entend, et ainsi le récit est doué d'une amplitude qui fait défaut à l'information ${ }^{79}$.

Une « amplitude » qui concerne la transmission des contes et leur variabilité, mais aussi le travail inconscient qui se fait après l'écoute chez chacun des auditeurs. « Narratrices nées », les conteuses de l'Heure du conte savent d'instinct que la vertu du récit, qui est affaire personnelle, a besoin d'un temps de latence. Ce silence d'après l'écoute, c'est l'amorce de la rêverie telle que l'entend Gaston Bachelard ${ }^{80}$. Nous parlions plus haut de la rumination individuelle succédant au partage de l'écoute. En outre, nous dit un commentateur de G. Bachelard lorsqu'il évoque le chapitre 3 de son livre intitulé Les rêveries vers l'enfance:

Pas de rêverie sans pause, sans rupture, sans suspension du temps quotidien, du temps qui fige et cristallise notre mémoire et, partant, notre identité. La rêverie, en somme, repose sur la remise en mouvement des souvenirs « purs », clairs et bien assignés à leur décor formel ${ }^{81}$.

Nos bibliothécaires, sans avoir lu les ouvrages de W. Benjamin ni de G. Bachelard (tous deux postérieurs à leur entreprise), semblent avoir inventé un lieu où se déploierait cette sorte de rêverie créative :

79. Benjamin, 1992, p. 211.

80. En particulier La Poétique de la rêverie (2005).

81. Тнівочтот, p. 75. Il y a un Bachelard pédagogue, sans doute moins connu. 
La rêverie est cet état simple où l'œuvre prend d'elle-même ses convictions. Et c'est ainsi que pour bien des écrivains et poètes la liberté de la rêverie ouvre les voies à l'œuvre ${ }^{82}$.

Un lieu où seraient en quelque sorte contrecarrés les effets de l'éducation d'après « l'âge de raison », où l'on s'efforce de socialiser l'enfant et de lui apprendre l'objectivité ${ }^{83}$. Un lieu où serait librement disponible de quoi nourrir leurs rêveries, que ce soit par la lecture, l'écoute, les images visuelles : ces rêveries qui excluent toute intention didacticienne et qui mènent alors à la créativité. Ce lieu, Claire Huchet, Marguerite Gruny et Mathilde Leriche l'ont créé et l'ont soutenu héroïquement durant quinze ans, s'interrogeant constamment, sans jamais perdre leur ardeur, voire leur audace.

\section{Bibliographie}

Bachelard Gaston, 2004 [1960], La Poétique de la rêverie, Presses universitaires de France (Coll. Quadrige), Paris, 183 p.

Benjamin Walter, 1992 [1936], « Le Narrateur » in Écrits français, présentés et introduits par Jean-Maurice Monnoyer, Gallimard, Paris, p. 206-229.

Bertherotte Philippe, 2008, La Tentation d'une île. Derrière les caméras de l'Île de la Tentation, éd. Jacob-Duvernet, Paris, p. 337.

Cone Bryant Sarah, 1911, Comment raconter des histoires à nos enfants et quelques histoires racontées, ouvrage traduit librement de l'anglais par Élisée Escande, Nathan, Paris, 304 p.

Cone Bryant Sarah, s.d., Comment raconter des histoires à nos enfants, $2^{\mathrm{e}}$ série, traduit librement de l'anglais par Elisée Escande, Librairie d'éducation Fernand Nathan, Paris.

82. BACHELARD, 2004, p. 137.

83. Ibid., p. 91. 


\section{CAHIERS DE LITTÉRATURE ORALE}

Gruny Marguerite, 1938, «L'Heure du conte. Quelques conseils sur l'art de raconter les histoires » in L'Éducation par la récréation, Ed. Berger-Levrault, Paris/Nancy, p. 70-86.

JaCQues Martine, 2013, «L'Île rose et La Colonie de Charles Vildrac : le modèle utopique en question dans la littérature pour la jeunesse des années vingt » in Strene, $\mathrm{n}^{\circ}$ 6, DOI : $10.4000 /$ strenae. 1075 .

LABRIE Vivian, 1978, La Tradition du conte populaire du Canada français : circonstances de la circulation et fonctionnement de la mémorisation, Université Paris V René-Descartes, Paris.

LERICHE Mathilde, 1934, « Les contes dans l'éducation des enfants de 6 à 11 ans, ou l'Heure du conte. Causerie faite à la "Nouvelle Éducation" » in Revue du livre et des bibliothéques, $\mathrm{n}^{\circ} 12$, p. 261-272.

Leriche Mathilde, 1956, On raconte, Armand Colin Bourrelier, Paris.

Patte Geneviève, 2000, « Hommage à Mathilde Leriche » in La Revue du livre et des enfants, $\mathrm{n}^{\circ} 191$, p. 15.

Patte Geneviève, 2005, «Dits et récits à la bibliothèque » in Cevin Évelyne (dir.), Conte en bibliothéque, Éditions du Cercle de la librairie, Paris, p. 107-135.

Piffault Olivier, 2006, « Postface » à Charles Vildrac, L'Île rose, Éditions Thierry Magnier, Paris, p. 157-161.

Tнiвоuтот Christian, « Les rêveries vers l'enfance dans l'œuvre de Gaston Bachelard : une lecture phénoménologique » in Recherches qualitatives, vol. 25(1), p. 62-87, http://www.recherche-qualitative.qc.ca/Revue.html.

Vildrac Charles, 1924, L'Île rose, illustrations d'Edy-Legrand, Tolmer, Paris, $147 \mathrm{p}$.

Vildrac Charles, 1930, La Colonie (L'île rose), illustré par Edy-Legrand, Albin Michel éditeur, Paris, $251 \mathrm{p}$. 


\section{Annexes}

«Une même lecture, deux publics ${ }^{84}$ »

PREMIER PUBLIC, 1927. - On est heureux de voir combien les enfants avaient bien suivi : Nils, le jars Martin, Akka, Smirr leur apparaissaient comme les personnages essentiels. - Ils sont impatients de savoir la suite. - La disparition du jars Martin provoque des exclamations inquiètes. - Succès. - Les détails pittoresques sur les coutumes les amusent et les intéressent. - Ils ne pensent pas que l'histoire de Nils aura jamais une fin, ils la vivent. Réclament parce que la séance est trop courte ... ! - Ils écoutent inlassablement. — L'histoire d'Akka et de l'aigle Gorgo les passionne. - Ils sont véritablement avec Nils et vivent avec lui. - La fin leur paraît infiniment triste. - Émotion très grande lorsque Nils redevenu grand fait ses adieux aux oies. - Ils disent après l'histoire : « On s'ennuie sans Nils ». On passe la soirée à regarder la grande édition du « Merveilleux voyage de Nils... » pour retrouver les épisodes racontés. Pour aimer ainsi Nils on a l'impression qu'ils ont vraiment senti la valeur de l'histoire, son sens profond ; que, comme Nils, ils ont appris quelque chose !

DEUXIEME PUBLIC, 1933. - Nils et les oies leur sont familiers mais il faut un peu simplifier : auditoire dans l'ensemble peu développé. - Tous aiment l'histoire et veulent savoir la suite, mais attention qui fléchirait facilement. Pourquoi ? on ne sait pas, peut être à cause de plusieurs nouveaux. - Beaucoup de coupures : il faut mettre très en relief les principaux événements et parfois beaucoup insister ou simplifier. - Impression qu'ils ne vont pas jusqu'au cœur de l'histoire. - Public dans l'ensemble un peu au dessous du niveau de Nils : les enfants sauf quelques-uns absolument enthousiasmés n'ont pas tiré tout le profit qu'ils auraient pu de Nils Holgersson.

84. LERICHE, 1934, p. 266-267. 


\section{Les contes de fées, ça c'est beau!!!!}

Résumé : Cet article tente de suivre la création et l'évolution d'une pratique inédite en France à cette époque tourmentée et pleine d'espoirs nouveaux, celle de l'entre-deux-guerres (1924-1939). Il s'agit de la pratique du contage aux enfants, dans un cadre lui aussi inédit : une bibliothèque qui leur est exclusivement consacrée, L'Heure joyeuse. Suivant l'exemple des États-Unis, les deux activités sont corollaires, l'une ne va pas sans l'autre. Les réactions des enfants à l'Heure du conte sont notées très soigneusement et analysées : il s'agit d'abord de susciter leur plaisir à l'écoute, voire leur enthousiasme, souvent attesté et qui s'inscrit dans le partage entre enfants et conteuse. On y remarque peu d'intention pédagogique manifeste, sinon parfois un retour spontané vers le livre dont a été tiré le conte dit. À un niveau plus profond, il s'agit d'amener l'enfant vers une rêverie poétique et créatrice au sens où l'entend G. Bachelard.

Mots-clefs : Heure du conte, pratiques de contage, tentation insulaire, invention d'une pratique.

\section{Fairy tales, it's beautiful!!!!}

Abstract: This article tries to follow the creation and evolution of an unprecedented practice in France at this time tourmented and full of hopes. This is the practice of children'storytelling in a new setting: a library entirely devoted to them: L'Heure joyeuse. Following the example of the United States, the two activities are corollary. Children's reactions to Story Hour are very carefully removed and analyzed. First of all, it's a question of stimulating their listening pleasure, even their enthusiasm which is often attested, and which is part of the sharing beetween children and storyteller. There is little obvious pedagogical intent if not sometimes a return to reading (return to the book from which the tale was taken). At a deep level, it's a question of bringing the child to a poetic and creative reverie in the sense of Gaston Bachelard.

Keywords: Story Hour, storytelling, island temptation, invention of a practic.

\section{Note sur l'auteur}

Nicole Belmont est directrice d'études à l'École des Hautes Études en sciences sociales et membre du Laboratoire d'anthropologie sociale (Collège de France). Ses recherches portent sur la littérature orale européenne et particulièrement les contes merveilleux. Elle a reçu le prix européen Walter Kahn 2017. 